\title{
Treatment Patterns, Overall Survival, and Total Healthcare Costs of Advanced Merkel Cell Carcinoma in the USA
}

\author{
Lotte Steuten ${ }^{1,4}$ (D) Vincent Garmo ${ }^{2,5} \cdot$ Hemant Phatak $^{2} \cdot$ Sean D. Sullivan ${ }^{3} \cdot$ Paul Nghiem $^{6} \cdot$ Scott D. Ramsey ${ }^{1}$
}

Published online: 28 June 2019

(c) The Author(s) 2019

\begin{abstract}
Background Merkel cell carcinoma (MCC) is a rare and aggressive type of cancer with poor outcomes.

Objective To describe treatment patterns, overall survival, and healthcare costs associated with advanced MCC (aMCC) using data from Medicare enrollees who received an aMCC diagnosis in the USA States between 2006 and 2013.

Methods Surveillance, Epidemiology, and End Results (SEER)-Medicare data from 2006 to 2013 were used to describe treatment patterns, 1- and 5-year overall survival, and total healthcare costs for the periods 12 months before aMCC diagnosis and $4-12$ months afterward in patients aged $\geq 65$ years.

Results We identified 257 patients with an aMCC diagnosis, of whom $51 \%$ had stage IIIb disease and $49 \%$ had stage IV. Within 4 months after diagnosis, $84 \%$ of patients $(n=216)$ received treatment; $45 \%(n=115)$ received surgery, $48 \%(n=124)$ radiation therapy, and 31\% $(n=80)$ chemotherapy. Second-line chemotherapy was administered in 33\% of patients $(n=26)$ receiving firstline chemotherapy. Median overall survival was 27 months in patients whose aMCC was diagnosed at stage IIIb and 12 months in patients whose aMCC was diagnosed at stage IV. Median total 12-month direct healthcare costs were US $\$ 48,006$ (25th-75th percentile range $=$ US $\$ 30,594-U S \$ 69,797$ ) per patient. Total costs were highest in patients receiving chemotherapy, either alone or combined with radiation and/or surgery (US $\$ 52,854 ; 25$ th-75th percentile range $=$ US $\$ 34,473-$ US $\$ 71,987$ ).

Conclusion Most patients with aMCC received initial treatment, including surgery, radiation, and/or chemotherapy, and approximately one-third of those receiving chemotherapy received second-line chemotherapy. Total 12-month direct healthcare costs were highest in patients who received chemotherapy alone or combined with radiation and/or surgery. These poor survival results and high treatment costs highlight the need for effective new aMCC therapies.
\end{abstract}

\section{Introduction}

Merkel cell carcinoma (MCC) is a rare and aggressive neuroendocrine tumor [1]. Studies based on the Surveillance, Epidemiology, and End Result (SEER) Program have shown that the estimated age-adjusted incidence rate for MCC was as low as $0.18-0.41$ per 100,000 population by the year 2006 [2]. Additional studies, however, have calculated a steep increase in MCC incidence rate over the last two decades

Parts of this study were previously presented at the 22nd Annual International Meeting of the International Society for Pharmaceutical Outcomes Research, Boston, MA, USA, 2017.

Electronic supplementary material The online version of this article (https://doi.org/10.1007/s40258-019-00492-5) contains supplementary material, which is available to authorized users.

Lotte Steuten

1steuten@ohe.org

Extended author information available on the last page of the article

\section{Key Points for Decision Makers}

The majority of patients with a diagnosis of aMCC received first-line therapy including surgery, radiation, and/ or chemotherapy. About one-third of patients given chemotherapy received a second-line chemotherapy treatment.

Median overall survival was 27 months for patients with stage IIIlb disease and 12 months for patients with stage IV disease.

Total direct healthcare costs quadrupled in the first 4 months after aMCC diagnosis and continued to rise up to a 10 -fold increase 12 months after diagnosis compared to 12 months pre-diagnosis. Direct total healthcare costs were highest for patients receiving treatment that included chemotherapy.

Poor survival results and high treatment costs found in this study highlight the need for (cost-) effective new therapies for patients diagnosed with advanced MCC. 
$[3,4]$, up to an estimated 0.7 cases per 100,000 person-years in the year 2013 in the USA [5]. This is owing to both better pathologic diagnostic tools and an aging population, because MCC incidence increases exponentially with age [5]. The prognosis of MCC is very poor because this cancer grows rapidly and has a high risk for recurrence and early metastasis $[6,7]$.

Traditional treatment approaches generally included surgery and/or radiotherapy for localized and regional disease, and cytotoxic chemotherapy for advanced disease [8]. Chemotherapy regimens for MCC were mostly extrapolated from those used for small-cell lung cancer, which are rarely curative in advanced MCC (aMCC) and are associated with significant toxicity [9].

With the emergence of immunotherapies for the treatment of patients with MCC, it is helpful to quantify their potential impact on treatment patterns and patient outcomes compared with that of established therapies. Crucial to understanding the comparative value of any new treatment option is real-world data describing the current treatment patterns, outcomes, and costs of care. Such data are scarce for aMCC and cannot easily be extrapolated from the small clinical trials in this rare disease. This study therefore describes firstand second-line treatment patterns, explores variables that potentially impact overall survival (OS), and estimates total direct healthcare costs, using data from Medicare enrollees who received an aMCC diagnosis in the USA between 2006 and 2013.

\section{Methods}

\subsection{Setting and Study Population}

Our patient sample was drawn from the SEER-Medicare database. SEER-Medicare includes pathological, staging, healthcare utilization, cost, and survival information for more than $94 \%$ of Medicare enrollees who received a cancer diagnosis in the SEER regions. For this study, we evaluated patients whose MCC was diagnosed between 2006 and 2013. The study population was restricted to patients with aMCC (stage IIIB+) who were continuously enrolled in Medicare Part A and Part B for a period beginning 12 months prior to diagnosis through 4 months following diagnosis. To calculate post-diagnosis medication cost, we also required patients to be enrolled in Medicare Part D for 4 months after first treatment.

To capture treatments only for aMCC, we required that MCC was the first cancer diagnosis or there was a 2-year period between MCC diagnosis and previous cancer diagnosis and no treatment (chemotherapy or radiation) was received 1 year prior to MCC diagnosis.
Patients were also excluded from the analyses if they had an unknown month of diagnosis, their diagnosis was made at autopsy or by death certificate, they had died within 4 months after receiving the MCC diagnosis, or if they were $<65$ years of age and had qualified for Medicare based on disability and/or having end-stage renal disease. We also excluded patients enrolled in Health Maintenance Organizations because claims data to track their treatment and other healthcare use were unavailable.

\subsection{Defining Lines of Therapy and Treatment Patterns}

First-line treatment is defined as the first claim(s) indicating use of surgery, radiation, and/or chemotherapy drugs/administration and was identified using relevant Healthcare Common Procedure Coding System (HCPCS), Revenue Center, and ICD-9-CM Volume 3 procedure codes from the date of MCC diagnosis to 4 months after. We followed advice provided by academic clinicians with expertise in treating MCC when determining the most salient treatments of interest for second-line chemotherapy (see Online Resource 1). Typically, a single cycle of first-line chemotherapy lasts 3 weeks, and patients can receive two to six cycles before switching to other chemotherapy drugs. Second-line treatment was defined as a claim for a different chemotherapeutic agent filed at least 6 weeks after the start of the first-line treatment, coinciding with or shortly after discontinuation of the first-line agent.

\subsection{Data Analysis}

\subsubsection{Overall Survival}

One-year and 5-year cumulative proportions of patients alive were analyzed using Kaplan-Meier estimators, stratified by type of first-line treatment received. Variables potentially impacting OS were analyzed using multivariable Cox regression with survival (in months) as a dependent variable and age at diagnosis, age-adjusted Charlson Comorbidity Index score, sex, and median zip code-level income as independent variables. The latter were selected based on data availability and expert opinion.

\subsubsection{Cost of Care}

Median per-patient total direct healthcare costs were calculated, including diagnostics and imaging, treatment procedures, inpatient and outpatient visits, hospice, home healthcare, and durable equipment for any condition. Total cost of care was measured as the total reimbursements for all claim lines. We calculated median total cost for the pretreatment period, defined as 12 months prior to treatment initialization, 
and median total costs at 4 and 12 months after the treatment period for the patients who survived and were followed up until then. As an indicator for healthcare resource utilization, we report the total number of patients with claims, the total number of claims per cost category, and the median (range) number of claims per patient for those who had at least one claim in that cost category.

We also analyzed median total costs by treatment setting, distinguishing inpatient hospitalization, outpatient visits, hospice, home healthcare, and other (including carrier and durable medical equipment) 12 months prior to treatment initialization and 4 months after treatment initialization. Total 12-month post-treatment initialization costs are reported as total median costs per patient by initial treatment received and categorized as no treatment, treatment without chemotherapy, and treatment with chemotherapy. The US Consumer Price Index was used to inflate costs to 2019 US dollars.

\section{Results}

\subsection{Patient Characteristics}

We identified 257 patients with aMCC, of whom 51\% $(n=131)$ had their disease diagnosed at stage IIIb and $49 \%$ $(n=126)$ had their disease diagnosed at stage IV. The average age of the population was close to 80 years, and the majority of patients were White males living in metropolitan areas, with median zip code-level income $>$ US\$60k and a median score of 1 on the Charlson Comorbidity Index (see Table 1).

\subsection{Treatment Patterns}

Within 4 months after diagnosis, $84 \%$ of patients $(n=216)$ received treatment. Among patients with stage IIIb MCC, the combination of surgery and radiation treatment was the most common initial treatment $(24 \%[n=31])$. Initial treatments involving chemotherapy were least common $(20 \%[n=26])$. In patients with stage IV disease, treatments including chemotherapy were the most common initial treatment modality (43\% [ $n=54]$ ) (see Table 1$)$.

Of 80 patients receiving first-line chemotherapy, $33 \%$ $(n=26)$ received a stage IIIb MCC diagnosis and $67 \%$ $(n=54)$ received a stage IV MCC diagnosis. The most frequently used first-line chemotherapies in patients with stage IIIb and stage IV disease were platinum-based therapies $(65 \%[n=17]$ and $63 \%[n=34]$, respectively). Thirtythree percent $(n=26)$ of the 80 patients who received firstline chemotherapy received second-line chemotherapy, which included a wide range of therapeutic agents and
Table 1 Patient characteristics and treatment pattern in the analytic cohort by stage of disease at diagnosis

\begin{tabular}{|c|c|c|}
\hline & \multicolumn{2}{|c|}{$\operatorname{All~aMCC~}^{\mathrm{a}}(N=257)$} \\
\hline & $\begin{array}{l}\text { Stage IIIB } \\
(n=131)\end{array}$ & $\begin{array}{l}\text { Stage IV } \\
(n=126)\end{array}$ \\
\hline \multicolumn{3}{|l|}{ Patient characteristic } \\
\hline Age, years, mean (SD) & $79.6(7.5)$ & $79.9(7.8)$ \\
\hline Male, $n(\%)$ & $86(66)$ & $91(72)$ \\
\hline White, $n(\%)$ & $126(96)$ & $118(94)$ \\
\hline \multicolumn{3}{|l|}{ Residence, $n(\%)$} \\
\hline Large metro and metro & $111(85)$ & $108(86)$ \\
\hline $\begin{array}{l}\text { Urban, less urban, and } \\
\text { rural }\end{array}$ & $20(15)$ & $18(14)$ \\
\hline $\begin{array}{l}\text { Residence zip code-level } \\
\text { annual median income, } \\
\text { mean (SD), US\$ }\end{array}$ & $67,937(26,373)$ & $64,217(25,386)$ \\
\hline \multicolumn{3}{|c|}{ Charlson Comorbidity Index, $n(\%)$} \\
\hline 0 & $60(46)$ & $55(44)$ \\
\hline 1 & $30(23)$ & $32(25)$ \\
\hline $2+$ & $41(31)$ & $39(31)$ \\
\hline \multicolumn{3}{|l|}{ Treatment pattern } \\
\hline $\begin{array}{l}\text { First-line treatment } \\
\text { received, } n(\%)\end{array}$ & $111(85)$ & $105(83)$ \\
\hline \multicolumn{3}{|c|}{ If treated, type of first-line treatment included, $n(\%)$} \\
\hline Surgery & $69(53)$ & $46(37)$ \\
\hline Radiation & $71(54)$ & $53(42)$ \\
\hline Chemotherapy & $26(20)$ & $54(43)$ \\
\hline \multicolumn{3}{|c|}{ Initial treatment combination, $n(\%)$} \\
\hline No treatment & $20(15)$ & $21(17)$ \\
\hline Surgery only & $28(21)$ & $19(15)$ \\
\hline Radiation only & $26(20)$ & $18(14)$ \\
\hline Surgery and radiation & $31(24)$ & $14(11)$ \\
\hline $\begin{array}{l}\text { Treatment including } \\
\text { chemotherapy }\end{array}$ & $26(20)$ & $54(43)$ \\
\hline
\end{tabular}

$a M C C$ advanced Merkel cell carcinoma, $S D$ standard deviation ${ }^{\text {a }}$ Based on American Joint Committee on Cancer Stage group (6th and 7th edition); derived Surveillance, Epidemiology, and End Results (SEER) Summary Stage (SS) 2000 values are used (derived SS 2000 distant $=$ stage 4 )

combinations, such as cisplatin, carboplatin, etoposide, irinotecan, cyclophosphamide, vincristine, and doxorubicin.

\subsection{Overall Survival}

The cumulative proportion of patients surviving at 1 year was $71 \%$ and $48 \%$ for those receiving a diagnosis of stage IIIb and stage IV disease, respectively; at 5 years, survival was $37 \%$ and $16 \%$, respectively (see Fig. 1). Median OS was 27 months for patients receiving an MCC diagnosis at stage IIIb and 12 months for those receiving a diagnosis at stage IV (see Fig. 1). There was no significant difference in 


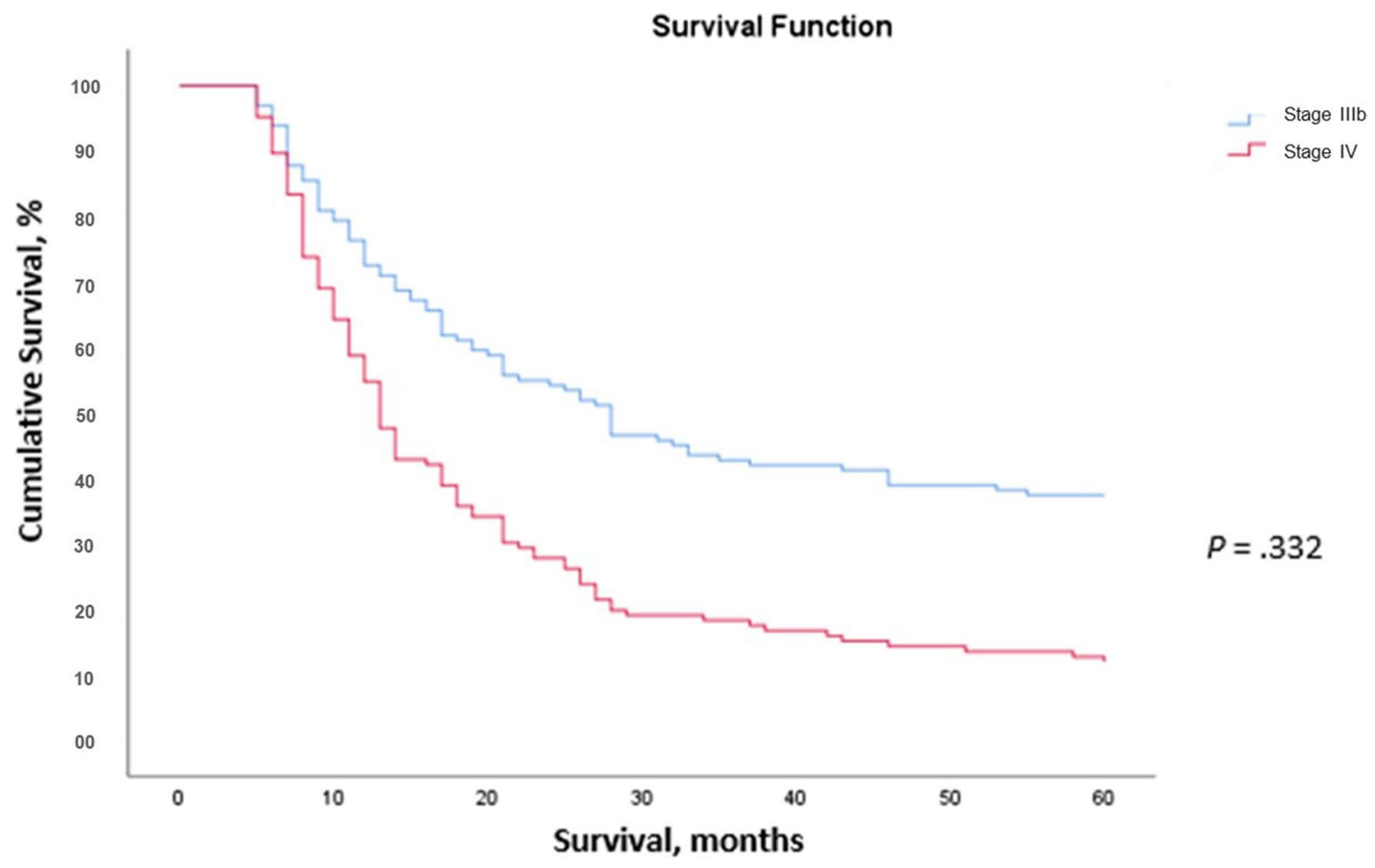

Fig. 1 Overall survival, stratified by stage IIIb and stage IV disease at diagnosis. No deaths occurred in the first 4 months due to the inclusion criteria of this study requiring at least 4 months of follow-up after an advanced Merkel cell carcinoma diagnosis

OS between patients receiving no treatment, patients treated with chemotherapy, or patients treated without chemotherapy (see Online Resource 2).

In patients whose MCC was diagnosed at stage IV, age $\leq 70$ years (hazard ratio (HR): 0.419 [95\% confidence interval (CI), 0.210-0835]; $P=0.013$ ), Charlson Comorbidity index $<1$ (HR: 0.503 [95\% CI, 0.315-0.803]; $P=0.004$ ) and median zip code-level income $>$ USUS $\$ 30,000$ per annum (HR: 0.248 [95\% CI, 0.104-0.590]; $P=0.024$ ) were associated with statistically significant better OS. Sex did not appear to be associated with OS. In patients whose MCC was diagnosed at stage IIIb, none of the variables reached statistical significance (see Online Resource 3 for details).

\subsection{Direct Healthcare Costs}

Patients receiving a diagnosis of aMCC have a median total direct healthcare cost of US\$5054 in the year preceding diagnosis (25th-75th percentile range: US\$1820-US\$17,375). The majority of patients had outpatient visits $(n=204)$ and laboratory tests done $(n=137)$, and nearly half of the patients ( $n=121$ ) had imaging (e.g., a positron emission tomography [PET], computed tomography [CT], or magnetic resonance imaging [MRI] scan). In addition, the majority ( $n=251$ ) of patients had fee-for-service claims for noninstitutional providers, such as physicians, physician assistants, clinical social workers, and nurse practitioners; and claims for services provided by freestanding facilities such as independent clinical laboratories, ambulance providers, and freestanding ambulatory surgical centers. Few patients were hospitalized $(n=63)$ or claimed home healthcare $(n=44)$ prior to diagnosis (see Table 2$)$.

During the 4 months after treatment initialization, the total direct healthcare costs per patient was a median of US\$22,399 (25th-75th percentile range: US\$15,528-US\$33,532). The majority of patients had claims for laboratory tests $(n=243)$ and imaging $(n=227)$, treatment monitoring (ranging from $n=148$ for a dermatology specialist to $n=253$ for a specialist other than an oncologist or dermatologist or a primary-care physician), and outpatient visits $(n=242)$. About $40 \%$ of patients $(n=112)$ were admitted to hospital, most $(n=99)$ for cancer-related reasons.

Median per-patient costs for MCC-related treatment were relatively low for surgical procedures (US\$1122 [25th-75th percentile range: US\$429-US\$2598) and chemotherapy (US\$1846 [25th-75th percentile range: US\$995-US\$4461) compared with radiation therapy (US\$7937 [25th-75th percentile range: US\$5597-US\$11,731]). Cancer-related inpatient hospitalizations were estimated to cost a median of US\$12,505 (25th-75th percentile range: US\$7192-US\$21,092) per patient. Approximately half of hospitalized patients were admitted through the emergency department (ED) (see Table 2). 
Table 2 Total direct healthcare costs, 12 months before and 4 months after treatment initialization

\begin{tabular}{|c|c|c|c|}
\hline Category & $\begin{array}{l}\text { No. of patients with claims } \\
\text { (total no. of claims) }\end{array}$ & $\begin{array}{l}\text { Median (range) no. of claims per } \\
\text { patient with } \geq 1 \text { claim }\end{array}$ & $\begin{array}{l}\text { Median cost per patient, } \\
\text { US\$ ( } 25 \text { th- } 75 \text { th } \% \\
\text { range) }\end{array}$ \\
\hline \multicolumn{4}{|c|}{ Total direct healthcare costs, 12 months prior to treatment initialization } \\
\hline Total cost & $257(23,807)$ & $58(1-501)$ & $5054(1820-17,375)$ \\
\hline \multicolumn{4}{|l|}{ Diagnostic procedures } \\
\hline Laboratory tests ${ }^{\mathrm{a}}$ & $137(843)$ & $19(1-400)$ & $626(190-1333)$ \\
\hline Imaging (PET, CT, PETCT, MRI) & $121(456)$ & $30(1-458)$ & $634(323-1399)$ \\
\hline Inpatient hospitalization & $63(139)$ & $1(1-305)$ & $18,446(7307-38,578)$ \\
\hline Admitted through ED & $43(139)$ & $1(1-305)$ & $9067(6049-19,100)$ \\
\hline Outpatient visits & $204(1257)$ & $5(1-32)$ & $893(278-2717)$ \\
\hline Carrier $^{\mathrm{b}}$ & $251(21,231)$ & $50(1-467)$ & $3471(1455-7249)$ \\
\hline Hospice & - & - & - \\
\hline Home healthcare & $44(72)$ & $1(1-132)$ & $3561(2871-6451)$ \\
\hline DME & $100(1102)$ & $64(1-465)$ & $359(122-986)$ \\
\hline \multicolumn{4}{|c|}{ Total direct healthcare costs, 4 months after treatment initialization } \\
\hline Total cost & $257(28,733)$ & $62(1-478)$ & $22,399(15,528-33,532)$ \\
\hline \multicolumn{4}{|l|}{ Diagnostic procedures } \\
\hline Laboratory tests ${ }^{\mathrm{a}}$ & $243(1463)$ & $4(1-24)$ & $1621(789-3563)$ \\
\hline Imaging (PET, CT, PETCT, MRI) & $227(1007)$ & $3(1-19)$ & $1628(1112-2431)$ \\
\hline \multicolumn{4}{|l|}{ MCC-related treatment } \\
\hline Surgical procedures & $118(1794)$ & $1(1-4)$ & $1122(429-2598)$ \\
\hline Radiation therapy & $129(2282)$ & $15(1-79)$ & $7937(5597-11,731)$ \\
\hline Chemotherapy & $84(1220)$ & $13(1-49)$ & $1846(995-4461)$ \\
\hline \multicolumn{4}{|l|}{ Treatment monitoring } \\
\hline Oncology specialty & $224(9680)$ & $93(1-455)$ & $2363(854-5840)$ \\
\hline Dermatology specialty & 148 (959) & $19(1-248)$ & $389(199-891)$ \\
\hline Primary care physician & $225(2616)$ & $60(1-465)$ & $319(149-733)$ \\
\hline Other & $253(15,478)$ & $47(1-478$ & $4220(2488-6061)$ \\
\hline Medication ${ }^{\mathrm{c}, \mathrm{d}}$ & $122(13,110)$ & $86(1-341)$ & $903(347-1695)$ \\
\hline Inpatient hospitalization & $112(205)$ & $1(1-10)$ & $14,003(8466-27,413)$ \\
\hline Cancer-related hospitalization & $99(156)$ & $5(1-42)$ & $12,505(7192-21,092)$ \\
\hline Admitted through ED & $55(205)$ & $1(1-10)$ & $12,244(6261-21,536)$ \\
\hline Outpatient visits & $242(2023)$ & $56(1-460)$ & $5813(2384-11,016)$ \\
\hline Carrier $^{\mathrm{b}}$ & $254(25,741)$ & $2(1-15)$ & $7662(5375-12,853)$ \\
\hline Hospice and home health care & $91(136)$ & $2(1-15)$ & $11,129(9238-13,982)$ \\
\hline DME & $96(625)$ & $5(1-72)$ & $166(100-481)$ \\
\hline
\end{tabular}

$C T$ computed tomography, $D M E$ durable medical equipment, $E D$ emergency department, $M C C$ Merkel cell carcinoma, $M R I$ magnetic resonance imaging, PET positron emission tomography, PET-CT positron emission tomography-computed tomography

${ }^{a}$ Laboratory tests include biopsy, surgical pathology examination, and immunochemistry

${ }^{\mathrm{b}}$ Carrier file (Physician/Supplier Part B claims file) contains final action fee-for-service claims submitted on a Centers for Medicare and Medicaid Services 1500 claim form. Most of the claims are from non-institutional providers, such as physicians, physician assistants, clinical social workers, and nurse practitioners. Claims for other providers, such as from freestanding facilities, are also captured in the carrier file. Examples include independent clinical laboratories, ambulance providers, and freestanding ambulatory surgical centers

${ }^{\mathrm{c}}$ We used prescription date to define whether medication use started within the initial treatment period

${ }^{\mathrm{d}}$ Sum of claims for ingredient cost paid, dispensing fee paid, and total amount attributed to sales tax

The total direct healthcare costs for patients who were followed up until 12 months after treatment initialization, as stratified by the first line of treatment received, showed that median per patient cost in patients receiving no treatment $(n=16)$ were US $\$ 32,054$ (25th-75th percentile range: US $\$ 23,034-\mathrm{US} \$ 43,222)$. In patients receiving surgery and/or radiation but no chemotherapy $(n=42)$, the median per-patients costs total US\$46,855 (25th-75th 
percentile range: US\$27,580-US\$65,258); and in patients receiving chemotherapy, either as a standalone treatment or combined with radiation and/or surgery $(n=34)$, these perpatients costs are US\$52,854 (25th-75th percentile range: US\$34,473-US\$71,987).

\section{Discussion}

Using a nationally representative claims database for Medicare patients, we described treatment patterns in patients who received an aMCC diagnosis and estimated their total direct healthcare costs and OS. In addition, we explored variables potentially impacting OS. Our study showed that the majority of patients with aMCC received first-line treatment that included surgery, radiation, and/ or chemotherapy alone or in combination. Chemotherapy treatment was the least common treatment modality received by patients whose MCC was diagnosed at stage IIIb (20\%) and the most common in patients whose disease was diagnosed at stage IV (43\%). Thirty-three percent of patients receiving chemotherapy as first-line treatment also received second-line chemotherapy. The most common chemotherapeutic treatments were combinations of etoposide, cisplatin, and carboplatin.

In the year prior to receiving an aMCC diagnosis, patients' median total per-patient direct healthcare costs were US $\$ 5054$. Many patients had claims for outpatient visits, laboratory tests, and imaging prior to diagnosis, which likely reflects that the presentation of MCC can be challenging for physicians to recognize [10]. Despite the high mean age in this population, few patients had claims for hospitalization or home health prior to diagnosis of aMCC.

The 5-year OS estimates of $37 \%$ and $16 \%$ for patients who received their MCC diagnosis at stage IIIb and stage IV, respectively, reflect the traditional treatment patterns before the use of immunotherapies in aMCC and show poor prognosis for patients with advanced disease. While the population included in this analysis are older (by $\sim 5$ years), these results are similar to those of previous studies reporting MCC-specific 5-year survival of 39-42\% in patients with regional nodal disease and $0-18 \%$ in patients with metastatic disease $[11,12]$. The similarity of survival curves in patients who received treatment and those who did not resonates with findings from previous studies indicating that despite initial effectiveness of chemotherapy, as seen in the stage IIIb patient group (see Online Resource 2), traditional treatments rarely provide durable responses [13].

Total direct healthcare costs more than quadrupled in the first 4 months after an aMCC diagnosis (US\$22,399) and were nearly 10 -fold higher in patients followed up until 12 months after diagnosis than in the year prior (US\$48,006 vs. US\$5054). As expected, total direct healthcare costs were lowest in patients receiving no treatment and approximately 1.5 times higher for patients receiving a treatment (combination) including surgery, radiation, and/or chemotherapy. Cancer-related hospitalization costs (median US\$12,505) were an important component of the total direct healthcare costs and were accrued in nearly $40 \%$ of patients after diagnosis. Notably, more than $50 \%$ of cancer-related inpatient hospital admissions occurred through the ER. The top reasons for hospital admission, as analyzed by ICD-9 codes, were chemotherapy related, including acute kidney failure, anemia, dehydration, and gout. Previous studies also showed that ER visits were common among cancer patients [14, 15] and may have been potentially preventable with better outpatient management [16].

The poor survival among Medicare enrollees with advanced-stage disease highlights the need for more effective aMCC therapies. In March 2017, the United States Food and Drug Administration (FDA) granted accelerated approval of the immunotherapy drug avelumab for the treatment of patients 12 years and older with metastatic MCC. Avelumab is a programmed death-ligand 1 (PD-L1)-blocking human immunoglobulin G1 lambda monoclonal antibody. It is the first and, so far, the only FDA-approved product to treat this type of cancer [17]. The efficacy and safety of avelumab was studied in the phase 2 JAVELIN Merkel 200 trial. Part A of this trial showed that $33 \%$ of 88 previously-treated patients with metastatic MCC achieved objective response, with 93\% of these responses lasting for at least 6 months [18]. A preplanned interim analysis of this trial showed that $62 \%$ of the 29 treatment-naïve patients with metastatic MCC achieved objective response, with $83 \%$ of these responses lasting for at least 6 months [19]. Another recent study investigated the immunotherapy drug pembrolizumab (which was previously approved by the FDA for the treatment of other cancers) in 26 patients with metastatic MCC who had not received any prior systemic therapy. Of those patients, $56 \%$ had an objective response, with $86 \%$ of the responders having ongoing response at the last follow-up before publication, which was more durable than the chemotherapy responses [20]. Based on these data, pembrolizumab was listed as a treatment option for patients with metastatic MCC in the 2017 NCCN annual consensus guidelines. Other immunotherapies, including nivolumab and ipilimumab, have also been tested in clinical trials in aMCC [21]. In addition, there are several other immunotherapy approaches being investigated for MCC in clinical trials, including intratumoral injection approaches and infusion of immune cells ( $\mathrm{T}$ cells or natural killer cells) [13].

Although the results of these studies are very promising, the rapidly increasing costs of existing as well as novel anticancer drugs [22] such as immunotherapies has raised awareness of the importance of assessing their "value", 
generally defined as the amount of effectiveness (e.g., life years) gained per dollar spent [23]. Value assessment frameworks have been developed in recent years for systematically assessing the value of medical treatments to allow for comparison and for recommendations on value-based pricing [24]. Previous work comparing the results of different value frameworks in assessing an immunotherapy for a rare cancer shows that the choice of value framework influences the assessment because each framework uses different factors to measure value [25]. However, when new drugs are superior it is currently difficult for insurers to limit their availability based solely on costs, especially for Medicare and Medicaid insurers, when these new drugs are approved by the FDA to treat life-threatening diseases.

This study established a baseline estimate of real-world treatment effectiveness and the costs of care in patients aged $\geq 65$ years who received a diagnosis of aMCC with which care with novel aMCC treatments can be compared. When doing so, the limitations of this study should be considered. Even when considering the SEER-Medicare cohort over many years, very few patients with aMCC were identified, limiting the statistical power to identify risk factors for high cost or poor outcomes or to test for differences in survival between treatment groups. Factors that could have influenced treatment patterns as well as costs and outcomes of care, including performance status, radiologic scans, and patient preferences for treatment or no treatment, are not available from the SEER-Medicare database. Also, treatment data relied on claim codes and are therefore subject to unverifiable errors. The estimated total costs 12 months after treatment were derived from the relatively small sample of patients who were followed up that long. Further, the exploration of potential predictors for OS in an observational study may suffer from selection bias, which hampers their interpretation. In particular, we caution against directly comparing these results for patients whose disease was diagnosed at stage IIIb versus stage IV because the study requirement of at least 4 months of follow-up after an aMCC diagnosis had a bigger impact on the latter group. Finally, the results of this study are not generalizable to commercially insured patients.

In conclusion, this study showed that the majority of patients with a diagnosis of aMCC received first-line therapy that included surgery, radiation, and/or chemotherapy, and about one-third of patients given chemotherapy received a second-line chemotherapy treatment. Median OS was 27 months for patients with stage IIIb disease and 12 months for patients with stage IV disease. Total direct healthcare costs surged in the first 4 months after an aMCC diagnosis and continued to rise throughout the 12 months after diagnosis compared with the 12 months prior. Direct total healthcare costs were highest for patients receiving treatment that included chemotherapy. Cancer-related hospitalizations were an important component of total direct healthcare costs before and after diagnosis.

Acknowledgements The authors gratefully acknowledge Catherine Fedorenko and Qin Sun for their data analytic support to this study.

Author contributions All authors made substantial contributions to the conception or design of the work; or the acquisition, analysis, or interpretation of data for the work; drafted the work or revised it critically for important intellectual content; gave final approval of the version to be published; and agree to be accountable for all aspects of the work in ensuring that questions related to the accuracy or integrity of any part of the work are appropriately investigated and resolved.

Data availability statement The data that support the findings of this study are available from the Surveillance, Epidemiology and End Results (SEER)-MEDICARE program, but restrictions apply to the availability of these data, which were used under license for the current study, and so are not publicly available. Analytic details are, however, available from the authors upon reasonable request and with permission of SEER-MEDICARE.

\section{Compliance with Ethical Standards}

Funding The research was funded by an alliance between Merck KGaA, Darmstadt, Germany and Pfizer, Inc, New York, USA. Merck Serono Ltd, Feltham, United Kingdom is an affiliate of Merck KGaA, Darmstadt, Germany. Dr. Steuten reports grants from EMD Serono, a business of Merck KGaA (Darmstadt, Germany) \& Pfizer, during the conduct of the study. Dr. Garmo reports personal fees from EMD Serono, outside the submitted work. Dr. Phatak reports personal fees from EMD Serono, outside the submitted work. Dr. Sean Sullivan reports grants from University of Washington, during the conduct of the study. Dr. Nghiem reports grants and personal fees from Merck EMD Serono GmbH, grants from Bristol Myers Squibb, personal fees from Merck, Sharp \& Dohme, personal fees from Pfizer, Inc, personal fees from Sanofi, Genzyme and Regeneron, outside the submitted work. Dr. Ramsey reports grants from EMD Serono, during the conduct of the study; grants and personal fees from Bristol Myers Squibb, personal fees from AstraZeneca, grants and personal fees from Bayer, personal fees from Genentech, personal fees from Seattle Genetics, outside the submitted work.

Open Access This article is distributed under the terms of the Creative Commons Attribution-NonCommercial 4.0 International License (http://creativecommons.org/licenses/by-nc/4.0/), which permits any noncommercial use, distribution, and reproduction in any medium, provided you give appropriate credit to the original author(s) and the source, provide a link to the Creative Commons license, and indicate if changes were made.

\section{References}

1. Toker C. Trabecular carcinoma of the skin. Arch Dermatol. 1972;105:107-10.

2. Albores-Saavedra J, Batich K, Chable-Montero F, Sagy N, Schwartz AM, Henson DE. Merkel cell carcinoma demographics, morphology, and survival based on 3870 cases: a population based study. J Cutan Pathol. 2010;37:20-7.

3. Becker JC. Merkel cell carcinoma. Ann Oncol. 2010;21:vii81-5. 
4. Tadmor T, Aviv A, Polliack A. Merkel cell carcinoma, chronic lymphocytic leukemia and other lymphoproliferative disorders: an old bond with possible new viral ties. Ann Oncol. 2011;22:250-6.

5. Paulson KG, Park SY, Vandeven NA, et al. Merkel cell carcinoma: current US incidence and projected increases based on changing demographics. J Am Acad Dermatol. 2018;78:457-63.

6. Donepudi S, Deconti RC, Samlowski WE. Recent advances in the understanding of the genetics, etiology, and treatment of Merkel cell carcinoma. Semin Oncol. 2012;39:163-72.

7. Muñoz IP, Masferrer JP, Vegas JO, Montalvo MSM, Díaz RJ, Casas AMP. Merkel cell carcinoma from 2008 to 2012: reaching a new level of understanding. Cancer Treat Rev. 2013;39:421-9.

8. NCCN Clinical Practice Guidelines in Oncology. Merkel Cell Carcinoma. V2.2018. National Comprehensive Cancer Network 2018. https://www.nccn.org/professionals/physician_gls/pdf/mcc. pdf. Accessed 19 Oct 2018

9. Voog E, Biron P, Martin JP, Blay JY. Chemotherapy for patients with locally advanced or metastatic Merkel cell carcinoma. Cancer. 2000;85:2589-95.

10. Colunga A, Pulliam T, Nghiem P. Merkel cell carcinoma in the age of immunotherapy: facts and hopes. Clin Cancer Res. 2018;24:2035-43.

11. Lemos BD, Storer BE, Iyer JG, et al. Pathologic nodal evaluation improves prognostic accuracy in Merkel cell carcinoma: analysis of 5823 cases as the basis of the first consensus staging system. J Am Acad Dermatol. 2010;63:751-61.

12. Santamaria-Barria JA, Boland GM, Yeap BY, et al. Merkel cell carcinoma: 30-year experience from a single institution. Ann Surg Oncol. 2013;20:1365-73.

13. Miller NJ, Bhatia S, Parvathaneni U, Iyer JG, Nghiem P. Emerging and mechanism-based therapies for recurrent or metastatic Merkel cell carcinoma. Curr Treat Options Oncol. 2013;14:249-63.

14. Subramanian S. Impact of Medicaid copayments on patients with cancer: lessons for Medicaid expansion under health reform. Med Care. 2011;49:842-7.

15. Kolodziej M, Hoverman JR, Garey JS, et al. Benchmarks for value in cancer care: an analysis of a large commercial population. $\mathrm{J}$ Oncol Pract. 2011;7:301-6.
16. Panattoni L, Fedorenko C, Greenwood-Hickman MA, et al. Characterizing potentially preventable cancer- and chronic diseaserelated emergency department use in the year after treatment initiation: a regional study. J Oncol Pract. 2018;14:e176-85.

17. US Food and Drug Administration, 2017. https://www.fda.gov/ Drugs/InformationOnDrugs/ApprovedDrugs/ucm547965.htm. Accessed 18 Oct 2018.

18. Kaufman HL, Russell JS, Hamid O, et al. Updated efficacy of avelumab in patients with previously treated metastatic Merkel cell carcinoma after $\geq 1$ year of follow-up: JAVELIN Merkel 200, a phase 2 clinical trial. J Immunother Cancer. 2018;6:7.

19. D'Angelo SP, Russell J, Lebbé C, et al. Efficacy and safety of first-line avelumab treatment in patients with stage IV metastatic Merkel cell carcinoma: a preplanned interim analysis of a clinical trial. JAMA Oncol. 2018;4:e180077.

20. Nghiem PT, Bhatia S, Lipson EJ, et al. PD-1 blockade with pembrolizumab in advanced Merkel-cell carcinoma. N Engl J Med. 2016;374:2542-52.

21. National Institutes of Health, US National Library of Medicine, ClinicalTrials.gov. https://clinicaltrials.gov. Accessed 19 Oct 2018.

22. Howard DH, Bach PB, Berndt ER, Conti RM. Pricing in the market for anticancer drugs. J Econ Perspect. 2015;29:139-62.

23. Sanders GD, Neumann PJ, Basu A, et al. Recommendations for conduct, methodological practices, and reporting of cost-effectiveness analyses: second panel on cost-effectiveness in health and medicine. JAMA. 2016;316:1093-103.

24. Willke RJ, Neumann PJ, Garrison LP Jr, Ramsey SD. Review of recent US value frameworks - a health economics approach: an ISPOR Special Task Force report. Value Health. 2018;21:155-60.

25. Garmo V, Lanitis T, Ambavane A, Kongnakorn T, Phatak H. Comparison of value evaluations using DrugAbacus and traditional cost-effectiveness analysis for an immuno-oncology drug in an orphan indication. In: ISPOR 22nd Annual International Meeting; May 20-24, 2017; Boston, MA [abstract 71931].

\section{Affiliations}

\section{Lotte Steuten ${ }^{1,4}\left(\mathbb{D} \cdot\right.$ Vincent Garmo $^{2,5} \cdot$ Hemant Phatak $^{2} \cdot$ Sean D. Sullivan ${ }^{3} \cdot$ Paul Nghiem $^{6} \cdot$ Scott D. Ramsey ${ }^{1}$}

\author{
Vincent Garmo \\ vincent.garmo@gmail.com \\ Hemant Phatak \\ hemant.phatak@emdserono.com \\ Sean D. Sullivan \\ sdsull@uw.edu \\ Paul Nghiem \\ pnghiem@uw.edu \\ Scott D. Ramsey \\ sramsey@fredhutch.org
}

1 Hutchinson Institute for Cancer Outcomes Research, Fred Hutchinson Cancer Research Center, 1100 Fairview Ave. North, Mail Stop M3-B232, Seattle, WA 98109, USA
2 EMD Serono, 1 Technology Place, Rockland, MA 02370, USA

3 School of Pharmacy, University of Washington, 1959 Northeast Pacific Street, Campus Box: 357630, Seattle, WA 98195, USA

4 Present Address: The Office of Health Economics, 7th Floor Southside, 105 Victoria Street, London SW1E 6QT, UK

5 Present Address: Genentech, 1 DNA Way, South San Francisco, CA 94080, USA

6 Division of Dermatology, Department of Medicine, University of Washington, 850 Republican Street, Box 358050, Seattle, WA 98109, USA 